\title{
Study on Virtual Simulation Experiment System of Fire Escape and Emergency Evacuation
}

\author{
Wenqi Zeng ${ }^{1}$ and Fengtao $\mathrm{Hao}^{2, *}$ \\ ${ }^{1}$ Teachers' College of Beijing Union University \\ 2 Teachers' College of Beijing Union University \\ *Corresponding author. Email: sftwenqi@buu.edu.cn

\begin{abstract}
Once the fire accident happens, it will seriously threaten people's lives. So it is very important to learn how to deal with dangerous situation. However, it is impossible to directly acquire the experience of fire escape and emergency evacuation by repeatedly experiencing the real situation of fire accident. So it is difficult to carry out practical teaching of fire-related knowledge. In this paper, a virtual simulation experiment system is constructed to reproduce the virtual scenes of fire accident and establish the virtual environment for fire escape and evacuation drills. The system provides learners with an intuitive learning method and creates an immersive real experience environment, so as to enhance their ability to cope with emergencies.
\end{abstract}

Keywords: Virtual simulation, Fire evacuation, Experiment teaching, Open sharing.

\section{THE SIGNIFICANCE OF VIRTUAL SIMULATION EXPERIMENT OF FIRE ESCAPE AND EMERGENCY EVACUATION}

Once the fire accident happens, it will seriously threaten people's life. People need to know how to deal with fire hazards, learn to escape quickly and evacuate in an emergency. However, due to the particularity and danger of fire accidents, it is difficult for the public to gain practical learning experience through personal experience in real life. Therefore, it is of great practical significance to study and apply the corresponding virtual simulation experiment system.

The fire environment can be simulated and realized in the experimental system. Fire escape scene is usually difficult to reproduce in real practice, and due to the limitations of space, the on-site escape drills cannot be repeated for many times. What's more, the skills learned in the occasional escape drill may not translate into the right actions in a crisis situation, especially when you face a real fire.

Therefore, the self-rescue process of fire escape can be presented through virtual simulation technology, which can solve this problem well. In the virtual simulation system, complex building environments can be constructed, in which people are crowded and it is difficult to evacuate and escape in case of fire. The system can guide the experimenters to adapt to the unfamiliar environment quickly, protect their own "safety" and organize evacuation at the same time.

Also, relevant experiments can be set up for special experimenters. In addition to fire escape self-rescue, the virtual simulation experiment of emergency evacuation is mainly aimed at some specific occupation, such as teachers, security personnel, building administrators and so on. For example, it is more important and difficult for teachers to organize the students to evacuate than personal safety in a fire accident. However, in a real fire emergency evacuation drill, a large number of people are involved, so repeated training and drills are not allowed.

By adopting virtual simulation technology, the fire scene and crowd can be presented virtually, and the virtual environment of escape drill and emergency evacuation can be created, so as to create an immersive teaching and training environment and provide an intuitive and effective learning means for participants. The integration of virtual simulation technology and education can better meet the practical needs of science education curriculum, and it is also a comprehensive training of practical ability to deal with emergencies. 


\section{TECHNICAL ARCHITECTURE OF VIRTUAL SIMULATION EXPERIMENT SYSTEM FOR FIRE ESCAPE AND EMERGENCY EVACUATION}

The operation of the virtual simulation experiment projects for fire escape and emergency evacuation relies on the support of the open virtual simulation experiment teaching management platform. Based on computer simulation technology, multimedia technology and network technology, the platform adopts serviceoriented software architecture, integrates physical simulation, innovative design, intelligent guidance, automatic correction and teaching management. Therefore, it is a great virtual experimental teaching platform with good autonomy, strong interactivity and expansibility.

The specific experimental project is seamlessly connected with the management platform through the data interface to ensure that users can access the project through the browser at anytime and anywhere. Besides, various user-oriented functions provided by the platform are utilized to strengthen the open service capability of the experimental project, improve the open service effect and realize independent experiments.

The overall architecture of the virtual simulation experiment teaching management platform is as shown in Figure 1.

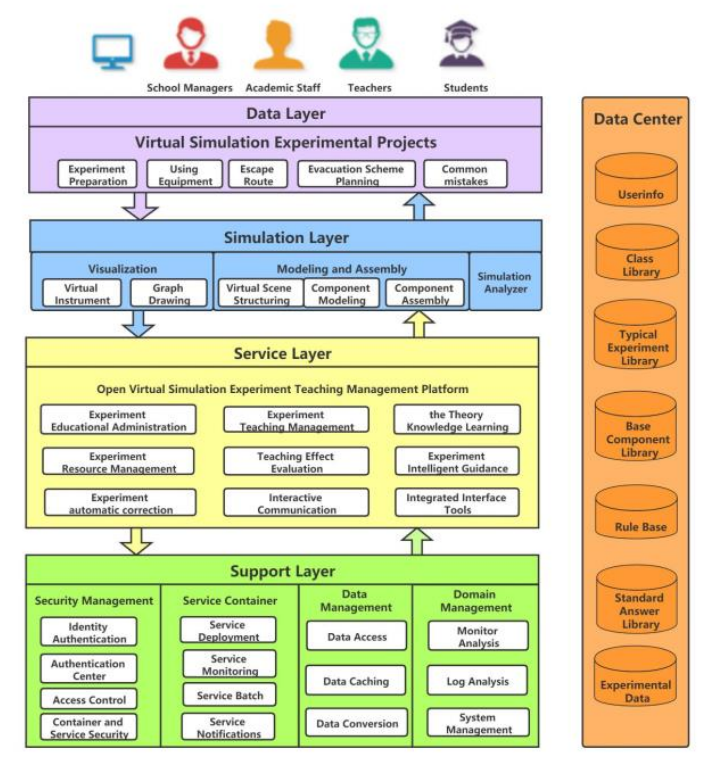

Figure 1 The overall architecture of the system

The overall architecture of the experimental system is divided into five layers, each of which provides services for the upper layer to complete the construction of specific virtual experimental teaching environment. The specific functions of each layer are as follows:

\subsection{Data Layer}

The virtual simulation experiments of fire escape and emergency evacuation involves various types of virtual experiment components and data. The system includes the basic elements library, experimental courses library, typical experiments library, standard answers library, rules library, experimental data and users' information of the virtual experiments and so on for the storage and management of the relevant data.

\subsection{Support Layer}

As the core framework of virtual simulation experiment system, the support layer is the basis for the normal open operation of experimental projects and is responsible for the operation, maintenance and management of the entire system. The supporting platform includes the following functional subsystems: security management, service container, data management, resource management and monitoring, domain management, inter-domain information service, etc.

\subsection{General Service Layer}

The general service layer provides the user interface of the open virtual simulation experiment system and the general support components of the virtual experiment teaching environment, so that users can quickly complete the virtual simulation experiment in the virtual experiment environment. General services include experiment educational administration, experiment teaching management, the theory knowledge learning, experiment resource management, intelligent guidance, interactive communication, automatic correction function, experiment report management, teaching effect evaluation, open and shared modules, etc. The general service layer also provides the relevant integrated interface tools, so that the system can integrate the third-party virtual experiment software to carry out unified management.

\subsection{Simulation Layer}

The simulation layer mainly creates models of experimental equipment, constructs the experiment scene, develops the virtual instrument, and provides the universal emulator. Finally, it provides the formatted output of experimental data for the upper layer.

\subsection{Application Layer}

Based on the services provided by the underlying layers, finally the virtual simulation experiment project of fire escape and emergency evacuation is taught and shared in the application layer. The application layer of the framework has good expansibility. According to 
teaching needs, teachers can design various typical experimental examples by using various tools provided by the service layer and corresponding equipment models provided by the simulation layer, and finally carry out experimental teaching for schools.

\section{PRINCIPLE AND PROCESS OF VIRTUAL SIMULATION EXPERIMENT OF FIRE ESCAPE AND EMERGENCY EVACUATION}

In the experiments of fire escape and emergency evacuation virtual simulation, the modern information technology is used to promote the reform of experiment teaching, which applied immersive, problem-based, interactive, autonomous and reflective teaching methods. So as to improve the ability of innovation, active learning and self-reflection for experiment participants.

\subsection{The Purpose of the Experiments}

(1) To virtualize the unrealizable real fire environment, intuitively feel the fearfulness and severity of fire, and conduct safety education for the experiment participants.

(2) To make the experiment participants experience the danger of fire firsthand and improve their safety awareness.

(3) To improve the ability of the experiment participants to respond to fire, rescue themselves and organize evacuation.

(4) To make the experiment participants master the use of fire extinguishers and other equipment, so as to reduce the experimental cost.

\subsection{The Teaching Knowledge Points of the Experiments}

(1) Personal safety knowledge and fire escape knowledge.

(2) Equipment operation knowledge.

(3) The design of escape route and the choice of escape method.

(4) The design of evacuation plan.

(5) Escape skills and evacuation under the condition of not serious fire.

(6) High-rise building escape skills and evacuation.

(7) Escape skills and evacuation in heavy smoke.

(8) Safety education of fire disaster

\subsection{The Implementation Process of the Experiments}

On the simulation platform, the virtual simulation experiment teaching will set up five parts including preview, demonstration, learning, assessment and report.

Preview Module: similar to experimental textbooks with experimental purposes, principles, operating steps, precautions, etc. Therefore, it is necessary to preview before conducting experiments.

Demonstration Module: including the learning video of the whole process of standard operation, which is convenient for the experiment participants to quickly understand the experimental content as a whole.

Learning Module: human-computer interaction can guide the experiment participants to complete the whole experiment step by step with the help of relevant prompts.

Assessment Module: conducting the operation test without any prompt and the system will give the score automatically after the assessment.

Report Module: After the completion of the assessment, the experimental report should be written, including the experimental purpose, principle, process, conclusion, and evaluation and suggestions for the experiment, and should be submitted to the teacher for review.

\subsection{Methods and Procedures of the Experiments}

The simulation training of fire escape and emergency evacuation are constructed through simulation experiments. The experimental project recreates the scene of fire scene by $3 \mathrm{~d}$ simulation technology and the experiment participants can carry out interactive operation in the whole scene to complete the experiment. The procedures of the experiments are shown in Figure2. 


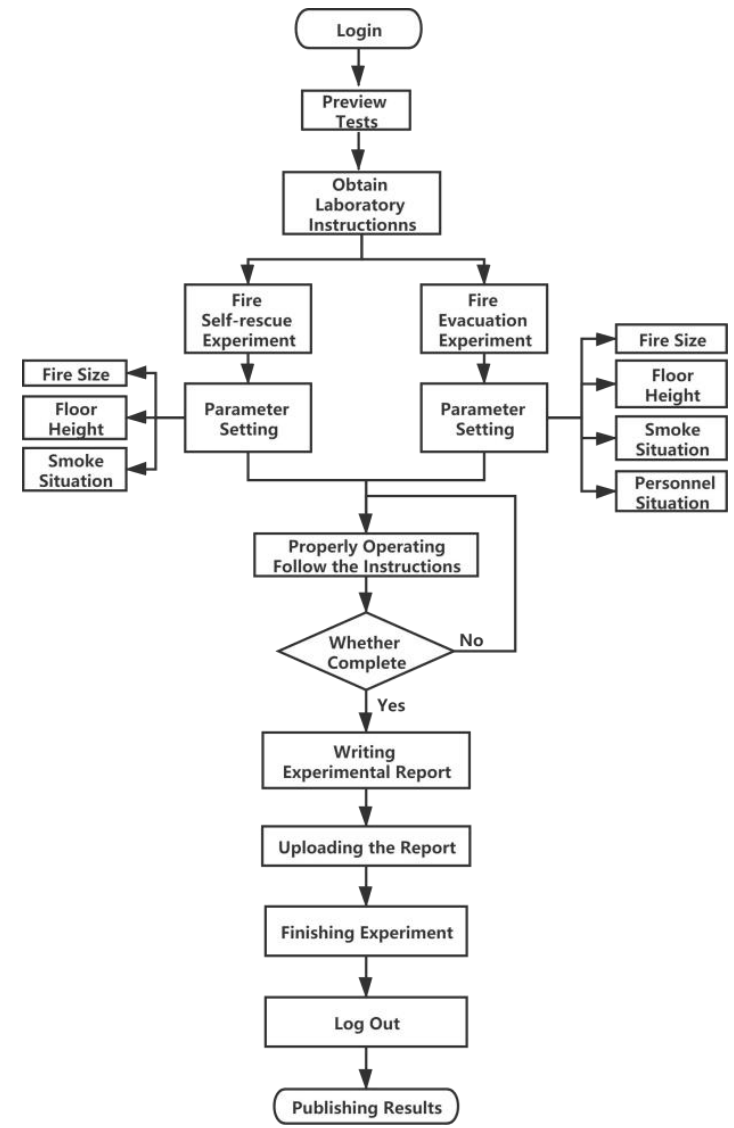

Figure 2 Procedures of the Experiments

\section{FEATURES OF EXPERIMENTAL TEACHINGPROJECTS}

\subsection{Diversified Teaching Methods}

The project follows the experimental teaching idea of teaching orientation, subject integration and innovative practice. Through the implementation of experiential immersion teaching method, the participants can master the escape skills in the event of fire and improve their emergency response ability, organization and coordination ability and comprehensive practice ability in the process of immersive experience, problem discrimination, interactive exercise, autonomous design and reflective evaluation.

\subsection{Obvious Teaching Effects}

This immersive teaching method can stimulate the learning interest of the experimental participants, deepen the knowledge experience of fire, improve the emergency response ability, and enhance the efficiency and ability of learning. The experimental method can also cultivate the habit of active learning and the ability to find, analyze, solve problems and think creatively.

\subsection{Evaluation System}

\subsubsection{Error Correction and Feedback}

In the normative practice of the project, the system will automatically prompt and correct the error when the operation is wrong. The experiment teacher can design the experiment independently, and the system automatically records the experiment process and operation steps throughout the whole process. The experiment participants can look back at their own operation records, prompting them to develop the habit of standardized practice and active thinking.

\subsubsection{Evaluation and Reflection}

In the assessment link, the system will automatically generate records and scores that can be traced back to the experimental process, so as to evaluate the operation of the experimental participants. The system carries out multi-dimensional assessment on the operation times, operation time, interactive operation points, etc. In addition, the theoretical knowledge of the experimental subjects is assessed through the experimental report, thus forming a comprehensive evaluation system that combines theory with practice, process and summative evaluation.

\subsection{The Extension and Development of Traditional Teaching}

Simulation system provides participants with high simulation of the virtual experiment environment, solving a series of problems, such as the risk of fire, lack of real environment, the limited experimental site, etc. which saves the cost of experiment teaching and extends the traditional laboratory with fixed class time to the network virtual laboratory and 24 hours online classroom in the air, so as to use modern information technology to extend the depth and breadth of the experimental content.

\section{CONCLUSION}

The Virtual Simulation Experiment System of Fire Escape and Emergency Evacuation reproduce the virtual scenes of fire accident and establish the virtual environment for fire escape and evacuation drills. So the system provides learners with an intuitive learning method and creates an immersive real experience environment, so as to enhance their ability to cope with emergencies. 


\section{REFERENCES}

[1] Fuquan Zhu, Liping Yang, The Application of Virtual Reality Technology in Fire Science, in: Science and Technology Innovation Herald, 2018, 15(09), pp:146-149.

[2] Dechuang Zhou, Study on Fire Scenario Computation and Simulation Based on Virtual Reality Platform, in: University of Science and Technology of China, 2009.

[3] Weiguo Wang, Construction Consideration and Suggestion of Virtual Simulation Experimental Teaching Center, in: Research and Exploration in Laboratory, 2013, 32(12), pp:5-8
[4] Yunming Zhang, Lei Chen, Fire Fighting and Rescue Training System Based on Virtual Reality Technology, in: Fire Science and Technology, 2010, 29 (11) , pp:996-998.

[5] Weiguo Wang, Jinhong $\mathrm{Hu}$, Hong Liu, Current Situation and Development of Virtual Simulation Experimental Teaching of Overseas Universities, in: Research and Exploration in Laboratory, 2015, 34(05), pp:214-219

[6] Ling Jiang, Xiaolu Liu, Yingqi Wang, A brief analysis in the current development of fire computer simulation technology, in: Fire Science and Technology, 2009, 28 (3) , pp:156-159 